\title{
Depression and the Overall Burden of Painful Joints: An Examination among Individuals Undergoing Hip and Knee Replacement for Osteoarthritis
}

\author{
Rajiv Gandhi, ${ }^{1}$ Michael G. Zywiel, ${ }^{1}$ Nizar N. Mahomed, ${ }^{1}$ and Anthony V. Perruccio ${ }^{1,2}$ \\ ${ }^{1}$ Division of Orthopaedic Surgery, Toronto Western Hospital, University of Toronto, 399 Bathurst Street 1E449, \\ Toronto, ON, Canada M5T 2S8 \\ ${ }^{2}$ Institute of Health Policy, Management \& Evaluation, University of Toronto, Toronto, ON, Canada
}

Correspondence should be addressed to Rajiv Gandhi; rajiv.gandhi@uhn.ca

Received 31 January 2015; Accepted 25 February 2015

Academic Editor: Henning Bliddal

Copyright (C) 2015 Rajiv Gandhi et al. This is an open access article distributed under the Creative Commons Attribution License, which permits unrestricted use, distribution, and reproduction in any medium, provided the original work is properly cited.

\begin{abstract}
The majority of patients with hip or knee osteoarthritis (OA) report one or more symptomatic joints apart from the one targeted for surgical care. Therefore, the purpose of the present study was to investigate the association between the burden of multiple symptomatic joints and self-reported depression in patients awaiting joint replacement for OA. Four hundred and seventy-five patients at a single centre were evaluated. Patients self-reported joints that were painful and/or symptomatic most days of the previous month on a homunculus, with nearly one-third of the sample reporting 6 or more painful joints. The prevalence of depression was $12.2 \%$ (58/475). When adjusted for age, sex, education level, hip or knee OA, body mass index, chronic condition count, and joint-specific WOMAC scores, each additional symptomatic joint was associated with a 19\% increased odds (odds ratio: 1.19 (95\% CI: 1.08, 1.31, $P<0.01)$ ) of self-reported depression. Individuals reporting 6 or more painful joints had 2.5-fold or greater odds of depression when compared to those patients whose symptoms were limited to the surgical joint. A focus on the surgical joint alone is likely to miss a potentially important determinant of postsurgical patient-reported outcomes in patients undergoing hip or knee replacement.
\end{abstract}

\section{Introduction}

Both depression and generally poorer mental well-being are more prevalent among individuals with chronic medical conditions when compared to those without chronic disease $[1,2]$. Additionally, poorer mental health status has been consistently shown to be associated with reduced overall health-related quality of life and with poorer outcomes of medical and/or surgical treatments $[3,4]$. Investigators have reported that pain associated with a range of chronic physical conditions is a significant contributing factor to increased rates of depression $[5,6]$.

Osteoarthritis (OA) is one of the most prevalent chronic medical conditions globally and is a leading cause of disability and health care utilization [7]. The cardinal symptom of $\mathrm{OA}$, as well as a leading factor for seeking medical care and surgical intervention, is pain [8]. Chronic pain is a strong predictor of clinical depression in this population, and both factors contribute to patient-reported functional disability associated with OA $[9,10]$. A number of studies have investigated the occurrence of depressed mood among OA clinical populations. One study, for instance, reported a $20 \%$ prevalence of depressive mood associated with OA within a primary care population [10], and similar rates have been documented among OA populations seeking specialist care $[11,12]$. Depressed mood has been linked with poorer functional outcomes of nonsurgical care in longitudinal, observational studies of knee OA [4] and with poorer pain and function scores and lower satisfaction, following interventions such as hip and knee replacement surgery for OA $[3,13,14]$. While several studies have examined the relationship between depressed mood and pain in OA and a number of studies have reported worse pain to be associated with more severe depression scores [10], the vast majority 
of investigations to date limited pain assessments to a single joint of interest. However, individuals with OA in one joint will often have symptomatic disease in one or more other joints, resulting in variable symptomatic disease burden that may not be appreciated when assessments are limited to a single joint [15-17].

Therefore, the purpose of this study was to investigate the association between the overall burden of symptomatic joints and self-reported depression among patients awaiting hip or knee replacement for end stage OA. We hypothesized that those with a greater burden of painful joints would report a greater prevalence of depression, independent of the magnitude of hip- or knee-specific pain in the surgical joint.

\section{Methods}

Patients with end stage hip or knee OA scheduled to undergo elective, primary total joint replacement were consecutively recruited from an academic hospital in Toronto, Canada, from April 2010 to November 2012. Eligibility criteria limited participants to those 18 years of age or older at the time of assessment and those who were able to read and understand English. Individuals with inflammatory arthritis or posttraumatic OA were excluded. Participants were mailed a health questionnaire consisting of a series of demographic and clinical questions and were asked to complete it two weeks prior to their scheduled surgical date. The study was approved by the University Health Network Research Ethics Board, and written informed consent was obtained from all study participants.

Demographic characteristics collected included age, sex, and level of education ( $\leq$ secondary school, > secondary school). Height and weight were abstracted from the clinical record of the preadmission assessment and used to calculate body mass index (BMI) in $\mathrm{kg} / \mathrm{m}^{2}$. The type of procedure planned (hip or knee replacement) was recorded for use as an indicator variable in the analyses described below.

Self-reported clinical data collected using the health questionnaire included medical comorbidities, number and location of symptomatic joints, and surgical joint-specific pain and function.

Individuals were asked to respond with yes or no to a series of questions asking whether they had one or more of a number of medical conditions. Additionally, for each question answered in the affirmative, patients were asked whether they were currently undergoing treatment for that condition [18]. Patient answers to the question of whether they had depression were used as the primary outcome of interest.

The number of locations of symptomatic joints was collected using a homunculus, on which participants were asked to graphically indicate all joints that had been painful/symptomatic for most days of the previous month. The graphical responses were examined and divided into discrete anatomical regions including neck, spine, shoulders, elbows, wrists, hands, hips, knees, ankles, and feet, with laterality noted as appropriate. Any marked joint was considered a symptomatic joint, consistent with the clinical criteria of the American College of Rheumatology [19, 20]. The number of joints indicated were summed to derive a painful joint score (ranging from 1 to 20), which formed the primary predictor of interest. The assessment was limited to joint pain only; no attempt was made to register the presence and/or distribution of more widespread, nonarticular, and/or myofascial pain. Similarly, patients were not specifically queried about the presence or absence of medical diagnoses such as fibromyalgia, which are characterized by widespread, nonarticular pain patterns.

Hip- or knee-specific pain and function was assessed using the Western Ontario McMaster University Osteoarthritis Index (WOMAC) pain and function subscales, respectively. The WOMAC index is among the most commonly used pain and function measures in lower-extremity OA populations, with demonstrated validity and responsiveness $[21,22]$. Possible scores range from 0 to 68 for the functional limitation scale and 0 to 20 for the pain scale, with higher scores indicating greater limitations/pain.

2.1. Statistical Analyses. Descriptive statistics were obtained for the overall sample. Bivariable analyses were performed to compare the distribution of variables between individuals who did and did not report depression using either the independent samples $t$-test or chi-square test, as appropriate.

Logistic regression modeling was used to investigate the association between painful joint count and reported depression (outcome), adjusted for age, sex (reference: female), level of education (reference: >high school), hip/knee OA group (reference: hip), BMI, comorbidity count, and WOMAC hip/knee-specific pain and functional limitation scores.

\section{Results}

Five hundred fifty-nine (559) individuals were eligible and enrolled in the study. Of these, 45 (8\%) withdrew from the study prior to returning the questionnaire or did not complete their questionnaires for other reasons. Five hundred and fourteen patients returned their questionnaires, of which 475 had complete data (207 hip, $268 \mathrm{knee})$. In total, data concerning $85 \%$ of eligible patients (475/559) were included in the final analysis.

The patients in the final study cohort had a mean age of 64.7 years, $57 \%$ of whom were female. The overall prevalence of patient-reported depression was $12.2 \%$ (58/475), with $91 \%$ of those patients (53/58) further reporting receiving treatment for this diagnosis at the time of questionnaire completion. Patients reporting depression were significantly more likely to be female, to have a higher BMI, higher comorbidity counts, and higher painful joint count (Table 1).

When adjusting for covariates using logistic regression modeling, an independent association was identified between the number of symptomatic joints and the likelihood of reporting depression (Table 2). Each numeric increase in symptomatic joint count was associated with $19 \%$ increased odds of reporting depression (odds ratio: 1.19 (95\% CI: 1.08, $1.31, P<0.01))$ (Table 2). 
TABLE 1: Comparison of demographic and clinical data between patients who did and did not report depression.

\begin{tabular}{|c|c|c|c|}
\hline & $\begin{array}{c}\text { Depression }=\text { yes } \\
(n=58)\end{array}$ & $\begin{array}{c}\text { Depression }=\text { no } \\
(n=417)\end{array}$ & $P$ value \\
\hline Mean age and SD (range) & $62.7 \pm 9.9(37$ to 86$)$ & $65.0 \pm 10.1$ (33 to 89$)$ & 0.105 \\
\hline Percentage of female patients & 82.5 & 53.5 & $<0.001$ \\
\hline Percentage of patients without any formal education beyond high school & 27.6 & 30.8 & 0.618 \\
\hline Percentage of patients awaiting knee (versus hip) replacement & 62.1 & 55.6 & 0.355 \\
\hline Mean body mass index in $\mathrm{kg} / \mathrm{m}^{2}$ and SD (range) & $33.5 \pm 8.7(21$ to 62$)$ & $29.9 \pm 6.3(19$ to 55$)$ & $<0.001$ \\
\hline Mean number of comorbidities and SD (range) & $2.7 \pm 1.7(0$ to 7$)$ & $1.7 \pm 1.3(0$ to 7$)$ & $<0.001$ \\
\hline Mean WOMAC pain score and SD in points (range) & $10.2 \pm 4.2(0$ to 20$)$ & $9.6 \pm 3.7(1$ to 18$)$ & 0.238 \\
\hline Mean WOMAC function score and SD in points (range) & $34.7 \pm 13.7(0$ to 68$)$ & $31.6 \pm 12.1(0$ to 62$)$ & 0.073 \\
\hline Mean number of painful joints and SD (range) & $7.3 \pm 4.7(1$ to 20$)$ & $4.1 \pm 3.1(1$ to 18$)$ & $<0.001$ \\
\hline
\end{tabular}

SD: standard deviation; WOMAC: Western Ontario and McMaster Universities Osteoarthritis Index.

TABLE 2: Results of logistic regression evaluating associations between symptomatic joint count and depression.

\begin{tabular}{|c|c|c|c|c|}
\hline Predictor variable & Reference value & Contrast value & Odds ratio & 95\% confidence interval \\
\hline Age in years & - & - & 0.96 & $0.93,0.99$ \\
\hline Sex & Male & Female & 4.24 & $1.74,10.35$ \\
\hline Education & $\begin{array}{l}\text { Some formal education } \\
\text { beyond high school }\end{array}$ & $\begin{array}{l}\text { No formal education } \\
\text { beyond high school }\end{array}$ & 1.05 & $0.50,2.23$ \\
\hline Surgical joint & Hip & Knee & 0.70 & $0.34,1.45$ \\
\hline Body mass index & - & - & 1.04 & $0.99,1.09$ \\
\hline Number of comorbidities & - & - & 1.50 & $1.15,1.85$ \\
\hline WOMAC pain score & - & - & 0.89 & $0.76,1.05$ \\
\hline WOMAC function score & - & - & 1.02 & $0.97,1.07$ \\
\hline Number of symptomatic joints & - & - & 1.19 & $1.08,1.31$ \\
\hline
\end{tabular}

WOMAC: Western Ontario and McMaster Universities Osteoarthritis Index.

\section{Discussion}

Depression has been associated with poorer treatment adherence in general clinical populations [23] and with worse health-related outcomes following both surgical and nonsurgical treatment in patients with OA specifically [3, 4]. As a result, an improved understanding of factors that contribute to depression in patients with $\mathrm{OA}$ and the identification of potentially modifiable correlates of depression may allow for the development of tailored treatment strategies that will positively impact outcomes in these patients. Despite the common presence of multiple symptomatic joints among individuals with $\mathrm{OA}$ in any one joint, studies to date have commonly limited their clinical assessment to the single joint of interest exclusively. It is likely that physicians and surgeons frequently similarly limit their clinical assessments as well. However, the results of the present study demonstrate that the presence of multiple other symptomatic joints in patients with end stage hip and knee OA is strongly associated with patient-reported depression.

Having been adjusted for several relevant factors, including age, sex, and other comorbid conditions, each numeric increase in painful joint count increased the odds of patientreported depression by almost $20 \%$. It is important to recognize that this relationship is cumulative and that in the present study patients could have up to 20 painful joints. Thus, individuals reporting 6 or more painful joints (encompassing one-third of the sample) had at least 2.5-fold increased odds of reporting depression compared to those who had symptoms in the surgical joint only. These findings speak to the importance of adopting a more holistic approach to the assessment of individuals with $\mathrm{OA}$ in the context of both clinical and surgical care and research activities [16]. While not specifically assessed in the present study, depression and depressed mood have been consistently shown to predict poorer patient-reported outcomes, including pain, activity limitations, and satisfaction with surgery $[13,14]$. Thus, the present results suggest that patients with OA and multiple painful joints may benefit from aggressive symptom management and potential psychological assessment [24].

Our findings are consistent with previous studies that have reported multisite body pain to be associated with incident depression among general medical and surgical populations $[25,26]$. A range of mediating links between chronic pain and depression have been proposed, ranging from altered neurotransmitter activity (norepinephrine, serotonin) within the central nervous system [27] to chronic sleep problems (chronic insomnia, unrefreshing sleep) [28]. The experience of chronic pain is reported to provoke a range of psychological reactions including helplessness, anxiety, depression, and anger [27]. Sleep-interrupting pain increases fatigue, while social relationships with friends and family 
may be adversely affected as well [28-30]. However, while depression, anxiety, and negative affect have been previously reported to be associated with magnitude of OA pain [31, 32], the causal direction of such relationships is difficult to discern. Unfortunately, the cross-sectional design of the present study prevents any assessment of directionality.

We acknowledge several potential limitations with the present study. First, our study sample was limited to individuals with late stage hip or knee OA associated with chronic joint pain. Second, while a longer duration of pain has been associated with a greater likelihood of depression [33], the duration of symptoms in the nonsurgical joints was not assessed in the patient questionnaire and thus was not considered in the analyses. Third, several factors may have influenced the prevalence of depression in the present study $(12 \%)$, which was lower than that reported in some other OA patient populations (20\%). Surgical candidacy is based on a number of factors that include medical fitness, and thus the present study cohort may not be representative of all OA patient populations. Furthermore, while many studies to date have stratified patients based on depressive symptoms identified using self-reported mood questionnaires, we defined depression as a self-reported condition without any attempt to confirm the presence of diagnostic criteria for this condition or any assessment of its chronicity. Nevertheless, with $91 \%$ of patients who self-reported having depression in the present study also reporting current treatment for it, it would appear that the large majority carried the formal diagnosis typically required before starting treatment. However, because the present study relied on self-reported depression, it is possible that some cases were missed because of imprecise recollection, unwillingness to report, or by a lack of clinical diagnosis. The extent to which missed cases relate to degree of painful joint burden is unknown. Fourth, 15\% of eligible patients either withdrew from the study or did not complete their questionnaire data. The lack of data for these patients precluded comparison with those who remained in the study, and thus the possibility of selection bias cannot be excluded. Fifth, no data were available concerning the presence and/or severity of widespread nonarticular pain patterns or the presence of central pain conditions such as fibromyalgia. Patients with fibromyalgia are known to sometimes report joint pain, and this condition has also been reported to be associated with poorer mental well-being. Thus, while the relationship between multiple symptomatic joints and central pain conditions in patients with known $\mathrm{OA}$ is not well understood, some of the findings of the present study may be explained by the concomitant presence of fibromyalgia or other similar conditions. As such, it would seem to be important to screen patient with OA for the presence of a comorbid central pain condition. Unfortunately, insufficient data were available to explore these relationships in the present study. Nevertheless, despite these limitations, we feel that the results of the present study do provide novel evidence for a clinically relevant association between the symptomatic joint burden in patients with OA and selfreported depression.

\section{Conclusions}

Given our finding that the presence of multiple painful joints in patients with established hip or knee OA is associated with an increased risk of prevalent depression, as well as likely depressed mood, an exclusive focus on the surgical joint alone is likely to miss a potentially important determinant of postsurgical patient-reported outcomes in this population. Consistent with other studies, we report that multiple painful joints are frequent in this population. While further study is needed to better understand the specific reasons for these associations, the authors suggest that clinicians and researchers consider the development of tailored treatment and management strategies for $\mathrm{OA}$ based on degree of multiple joint involvement, along with patient education, to address the unique characteristics of this distinct subgroup of patients.

\section{Abbreviations}

OA: Osteoarthritis

WOMAC: Western Ontario and McMaster

Universities Osteoarthritis Index.

\section{Ethical Approval}

REB approval was sought and granted for this study.

\section{Disclosure}

The paper was prepared wholly by the authors, without external and/or paid writing or editorial assistance.

\section{Conflict of Interests}

The following authors have received financial support from the following companies and/or organizations: Nizar N. Mahomed (Smith and Nephew, Bayer, Biomet, Stryker). None of the other authors have any financial competing interests to disclose. None of the authors have any nonfinancial competing interests to disclose.

\section{Authors' Contribution}

Study design was carried out by Rajiv Gandhi, Nizar N. Mahomed, and Anthony V. Perruccio. Data was gathered by Nizar N. Mahomed, Rajiv Gandhi, and Anthony V. Perruccio. Data analysis was done by Rajiv Gandhi and Michael G. Zywiel. Michael G. Zywiel and Rajiv Gandhi wrote the initial draft. Michael G. Zywiel, Nizar N. Mahomed, and Anthony V. Perruccio ensured the accuracy of data and analysis. All authors read and approved the final paper.

\section{Acknowledgments}

The present work was supported by internal divisional funding from the Arthritis Program, University Health Network, 
Toronto, Ontario, Canada. Study group: Arthritis Program, University Health Network.

\section{References}

[1] L. E. Egede, "Major depression in individuals with chronic medical disorders: prevalence, correlates and association with health resource utilization, lost productivity and functional disability," General Hospital Psychiatry, vol. 29, no. 5, pp. 409416, 2007.

[2] W. C. Jackson, "Assessing and managing pain and major depression with medical comorbidities.," The Journal of clinical psychiatry, vol. 74, no. 12, article e24, 2013.

[3] A. V. Perruccio, A. M. Davis, S. Hogg-Johnson, and E. M. Badley, "Importance of self-rated health and mental well-being in predicting health outcomes following total joint replacement surgery for osteoarthritis," Arthritis Care and Research (Hoboken), vol. 63, no. 7, pp. 973-981, 2011.

[4] L. Sharma, S. Cahue, J. Song, K. Hayes, Y.-C. Pai, and D. Dunlop, "Physical functioning over three years in knee osteoarthritis: role of psychosocial, local mechanical, and neuromuscular factors," Arthritis and Rheumatism, vol. 48, no. 12, pp. 33593370, 2003.

[5] M. M. Ohayon and A. F. Schatzberg, "Using chronic pain to predict depressive morbidity in the general population," Archives of General Psychiatry, vol. 60, no. 1, pp. 39-47, 2003.

[6] M. M. Ohayon and A. F. Schatzberg, "Chronic pain and major depressive disorder in the general population," Journal of Psychiatric Research, vol. 44, no. 7, pp. 454-461, 2010.

[7] V. Rabenda, C. Manette, R. Lemmens, A.-M. Mariani, N. Struvay, and J. Y. Reginster, "Direct and indirect costs attributable to osteoarthritis in active subjects," Journal of Rheumatology, vol. 33, no. 6, pp. 1152-1158, 2006.

[8] J. P. Clark, P. L. Hudak, G. A. Hawker et al., “The moving target: a qualitative study of elderly patients' decision-making regarding total joint replacement surgery," The Journal of Bone and Joint Surgery-American Volume, vol. 86, no. 7, pp. 1366-1374, 2004.

[9] M. J. Bair, R. L. Robinson, W. Katon, and K. Kroenke, "Depression and pain comorbidity: a literature review," Archives of Internal Medicine, vol. 163, no. 20, pp. 2433-2445, 2003.

[10] T. Rosemann, M. Backenstrass, K. Joest, A. Rosemann, J. Szecsenyi, and G. Laux, "Predictors of depression in a sample of 1,021 primary care patients with osteoarthritis," Arthritis Care \& Research, vol. 57, no. 3, pp. 415-422, 2007.

[11] B. C. Hanusch, D. B. O'Connor, P. Ions, A. Scott, and P. J. Gregg, "Effects of psychological distress and perceptions of illness on recovery from total knee replacement," Bone and Joint Journal, vol. 96, no. 2, pp. 210-216, 2014.

[12] E. A. Lingard and D. L. Riddle, "Impact of psychological distress on pain and function following knee arthroplasty," The Journal of Bone and Joint Surgery-American Volume, vol. 89, no. 6, pp. 1161-1169, 2007.

[13] J. Blackburn, A. Qureshi, R. Amirfeyz, and G. Bannister, "Does preoperative anxiety and depression predict satisfaction after total knee replacement?” Knee, vol. 19, no. 5, pp. 522-524, 2012.

[14] V. Brander, S. Gondek, E. Martin, and S. D. Stulberg, "Pain and depression influence outcome 5 years after knee replacement surgery," Clinical Orthopaedics and Related Research, no. 464, pp. 21-26, 2007.

[15] G. A. Hawker, E. M. Badley, C. M. Borkhoff et al., "Which patients are most likely to benefit from total joint arthroplasty?" Arthritis \& Rheumatism, vol. 65, no. 5, pp. 1243-1252, 2013.
[16] A. E. Nelson, M. W. Smith, Y. M. Golightly, and J. M. Jordan, "'Generalized osteoarthritis': a systematic review," Seminars in Arthritis \& Rheumatism, vol. 43, no. 6, pp. 713-720, 2014.

[17] A. V. Perruccio, J. D. Power, H. M. K. Evans et al., "Multiple joint involvement in total knee replacement for osteoarthritis: effects on patient-reported outcomes," Arthritis Care and Research, vol. 64, no. 6, pp. 838-846, 2012.

[18] O. Sangha, G. Stucki, M. H. Liang, A. H. Fossel, and J. N. Katz, "The Self-Administered Comorbidity Questionnaire: a new method to assess comorbidity for clinical and health services research," Arthritis Care and Research, vol. 49, no. 2, pp. 156163, 2003.

[19] R. Altman, G. Alarcón, D. Appelrouth et al., “The American college of rheumatology criteria for the classification and reporting of osteoarthritis of the hip," Arthritis and Rheumatism, vol. 34, no. 5, pp. 505-514, 1991.

[20] R. Altman, E. Asch, D. Bloch et al., "Development of criteria for the classification and reporting of osteoarthritis. Classification of osteoarthritis of the knee. Diagnostic and Therapeutic Criteria Committee of the American Rheumatism Association," Arthritis and Rheumatism, vol. 29, no. 8, pp. 1039-1049, 1986.

[21] N. Bellamy, W. W. Buchanan, C. H. Goldsmith, J. Campbell, and L. W. Stitt, "Validation study of WOMAC: a health status instrument for measuring clinically important patient relevant outcomes to antirheumatic drug therapy in patients with osteoarthritis of the hip or knee," The Journal of Rheumatology, vol. 15, no. 12, pp. 1833-1840, 1988.

[22] S. McConnell, P. Kolopack, and A. M. Davis, "The Western Ontario and McMaster Universities Osteoarthritis Index (WOMAC): a review of its utility and measurement properties," Arthritis Care and Research, vol. 45, no. 5, pp. 453-461, 2001.

[23] M. R. DiMatteo, H. S. Lepper, and T. W. Croghan, "Depression is a risk factor for noncompliance with medical treatment metaanalysis of the effects of anxiety and depression on patient adherence," Archives of Internal Medicine, vol. 160, no. 14, pp. 2101-2107, 2000.

[24] P. Creamer and M. C. Hochberg, "The relationship between psychosocial variables and pain reporting in osteoarthritis of the knee," Arthritis Care and Research, vol. 11, no. 1, pp. 60-65, 1998.

[25] D. A. Fishbain, R. Cutler, H. L. Rosomoff, and R. S. Rosomoff, "Chronic pain-associated depression: antecedent or consequence of chronic pain? A review," Clinical Journal of Pain, vol. 13, no. 2, pp. 116-137, 1997.

[26] M. M. J. G. Gerrits, P. van Oppen, H. W. J. van Marwijk, B. W. J. H. Penninx, and H. E. van der Horst, "Pain and the onset of depressive and anxiety disorders," Pain, vol. 155, no. 1, pp. 53-59, 2014.

[27] J. Dersh, P. B. Polatin, and R. J. Gatchel, "Chronic pain and psychopathology: research findings and theoretical considerations," Psychosomatic Medicine, vol. 64, no. 5, pp. 773-786, 2002.

[28] J. D. Power, E. M. Badley, M. R. French, A. J. Wall, and G. A. Hawker, "Fatigue in osteoarthritis: a qualitative study," $B M C$ Musculoskeletal Disorders, vol. 9, article 63, 2008.

[29] M. A. M. Gignac, C. L. Backman, A. M. Davis et al., "Understanding social role participation: what matters to people with arthritis?" Journal of Rheumatology, vol. 35, no. 8, pp. 1655-1663, 2008.

[30] G. A. Hawker, M. A. M. Gignac, E. Badley et al., "A longitudinal study to explain the pain-depression link in older adults with osteoarthritis," Arthritis Care and Research, vol. 63, no. 10, pp. 1382-1390, 2011. 
[31] P. Creamer, M. Lethbridge-Cejku, and M. C. Hochberg, "Determinants of pain severity in knee osteoarthritis: effect of demographic and psychosocial variables using 3 pain measures," The Journal of Rheumatology, vol. 26, no. 8, pp. 1785-1792, 1999.

[32] J. Dekker, P. Tola, G. Aufdemkampe, and M. Winckers, "Negative affect, pain and disability in osteoarthritis patients: the mediating role of muscle weakness," Behaviour Research and Therapy, vol. 31, no. 2, pp. 203-206, 1993.

[33] S. R. Currie and J. Wang, "Chronic back pain and major depression in the general Canadian population," Pain, vol. 107, no. 1-2, pp. 54-60, 2004. 


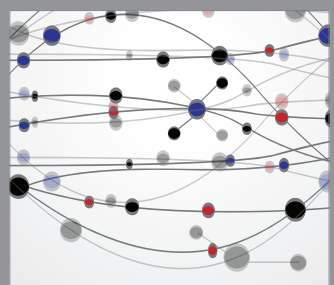

The Scientific World Journal
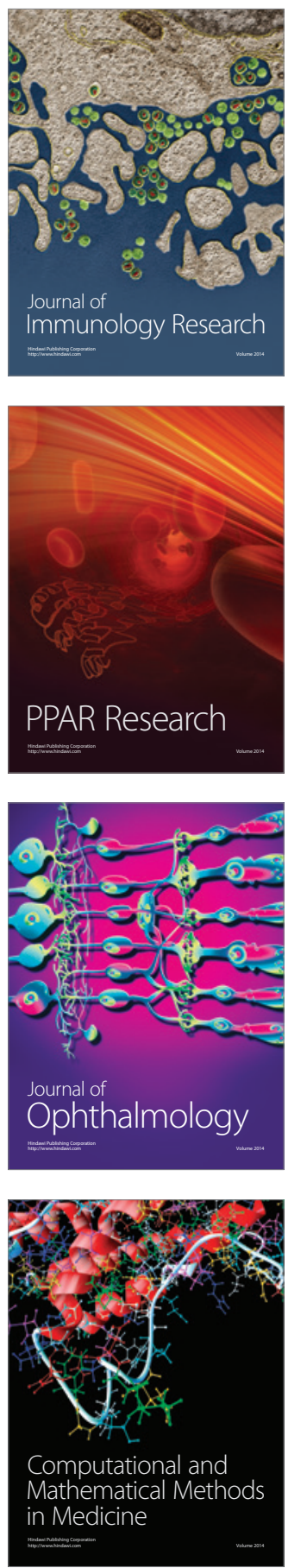

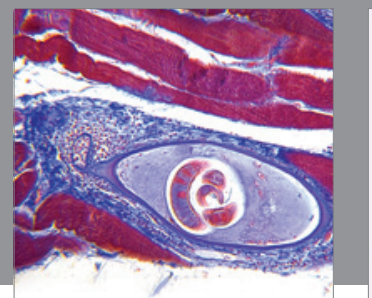

Gastroenterology

Research and Practice
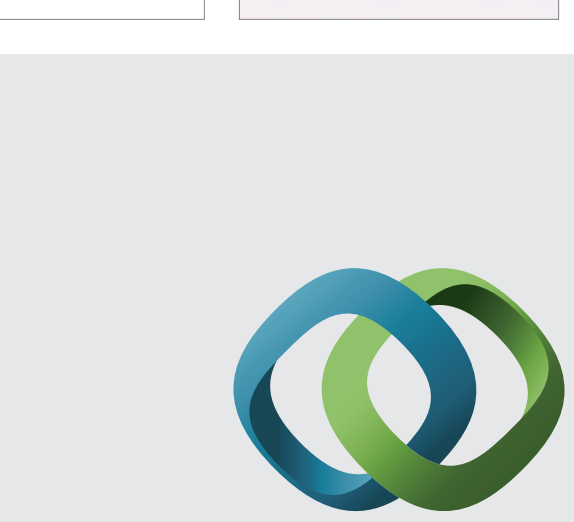

\section{Hindawi}

Submit your manuscripts at

http://www.hindawi.com
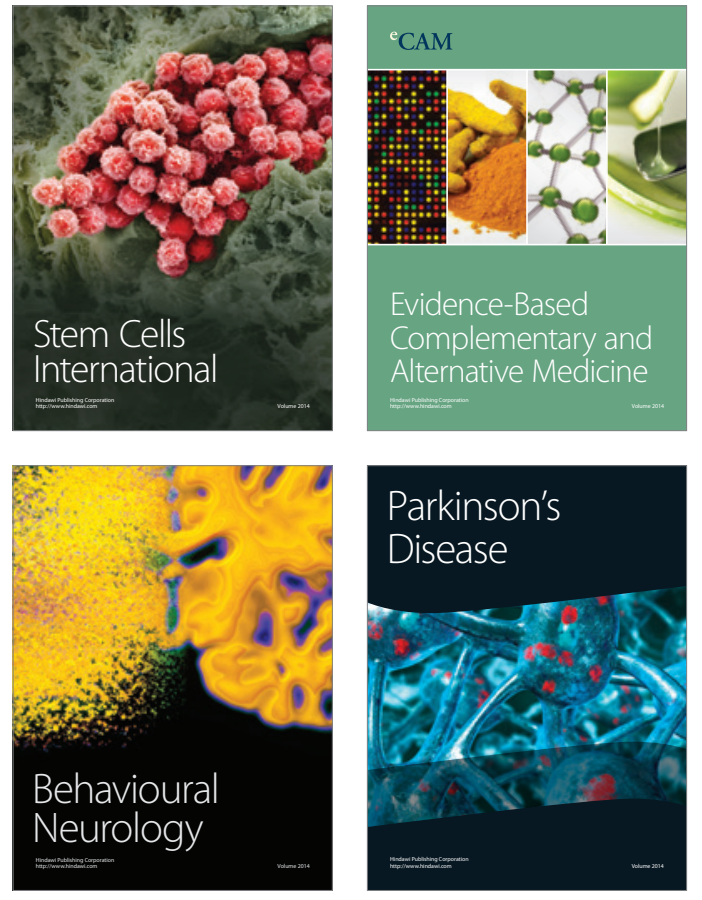
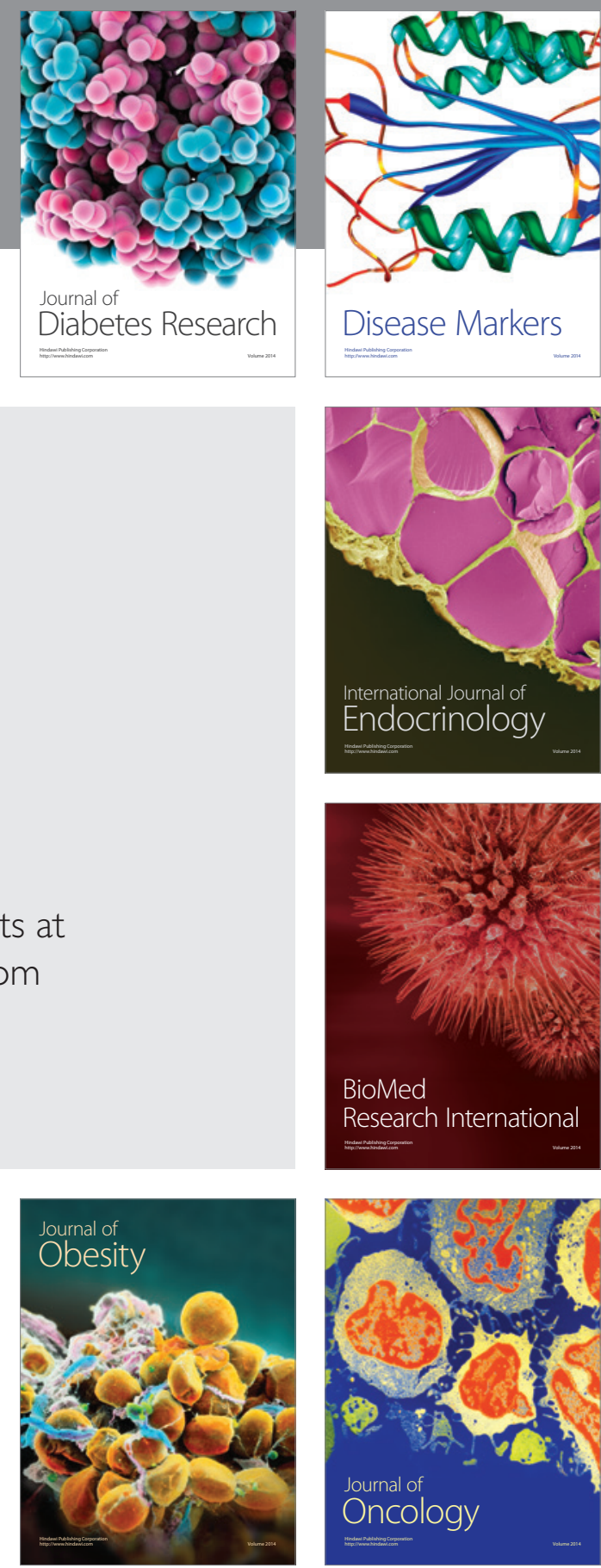

Disease Markers
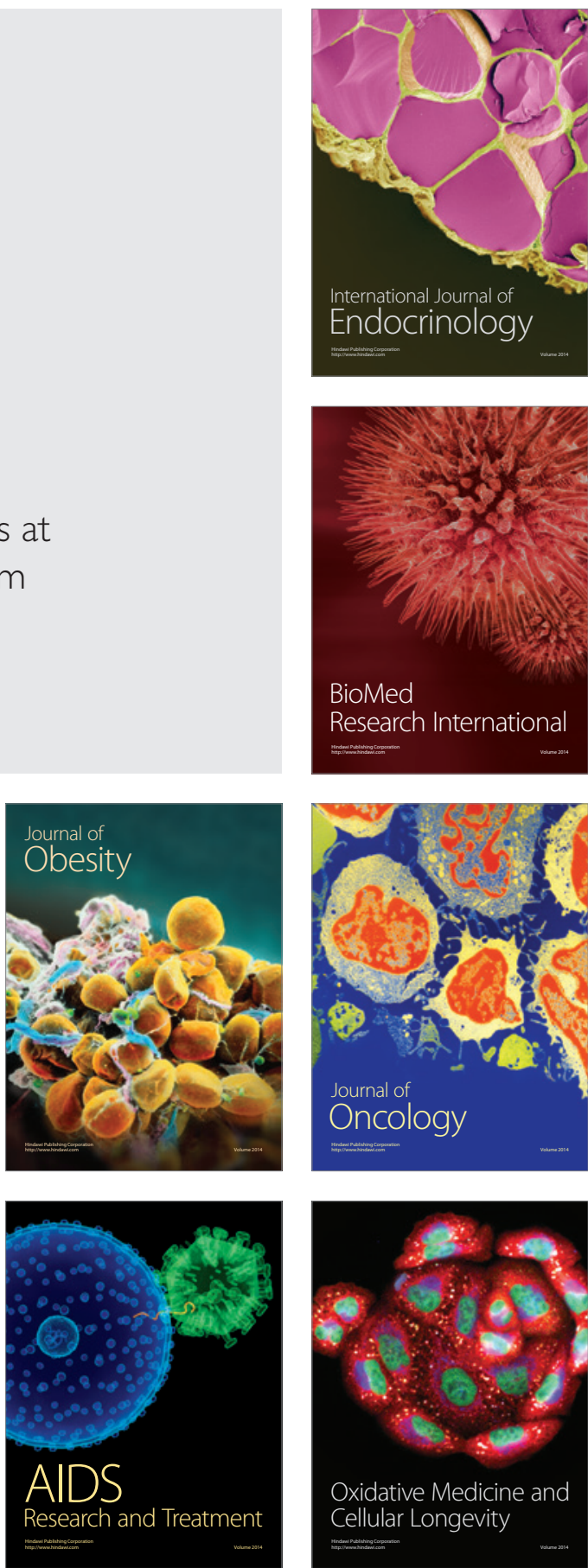